\title{
The Location Parameter Estimation of Spherically Distributions with Known Covariance Matrices
}

\author{
Hamid Karamikabir, Mahmoud Afshari* \\ Department of Statistics, Persian Gulf University, Bushehr, Iran
}

\begin{abstract}
This paper presents shrinkage estimators of the location parameter vector for spherically symmetric distributions. We suppose that the mean vector is non-negative constraint and the components of diagonal covariance matrix is known. We compared the present estimator with natural estimator by using risk function. We show that when the covariance matrices are known, under the balance error loss function, shrinkage estimator has the smaller risk than the natural estimator. Simulation results are provided to examine the shrinkage estimators.
\end{abstract}

Keywords Balance loss function, Spherical distribution, Risk function, Shrinkage estimator.

AMS 2010 subject classifications 60E05, 62F10, 62G05

DOI: $10.19139 /$ soic-2310-5070-710

\section{Introduction}

Shrinkage estimation is a method that a naïve or target estimator is improved, in some sense, by combining it with other information. Location parameter estimation is an important issue in the context of shrinkage estimation, specially when some components of location vector-parameter are restricted to be situated in a space.

Casella and Strawderman [4] and Bickel [1], studied the problem of location estimation under the mean constraint $|\theta| \leq m$ in the univariate normal distribution. Also Gatsonis, et al., [11] considered the Bayes estimator of the restricted normal mean under the same constraint. Kariya [16], Perron and Giri [26] and Marchand [18], proposed the best equivariant estimator of the mean $\theta$ in the spherical distribution under the local constraint $\|\theta\|=\left(\theta^{\prime} \theta\right)^{1 / 2}=\lambda_{0}$. Afterward [20] constructed a restricted estimator for the mean of the multivariate normal distribution when both mean vector $\mu$ and matrixcovariance $\Sigma$ were unknown; by adopting a constraint of the form $\mu^{\prime} \Sigma^{-1} \mu=\lambda_{0}$. Marchand and Giri [19] took the class of James-Stein type estimators and extended the estimation of the restricted mean in the class of variance mixture of normal distributions.

Fourdrinier and Ouassou [6] initiated the restricted estimation problem of the mean for the general spherical model, heuristically within the context of extra observation, by means of considering the spherically symmetric distributed observation $(X, U)$ around a vector of the form $(\theta, 0)$ with $\operatorname{dim} X=\operatorname{dim} \theta=p$ and $\operatorname{dim} U=\operatorname{dim}$ $0=k$. Wan et al. [30] obtained minimax and $\Gamma$-minimax estimators in the Poisson distribution when the parameter space was restricted by an interval, i.e., $\theta \in[0, \beta], \beta>0$. Again Fourdrinier et al. [7], interestingly considered the restricted estimation in the latter specified general spherical model, under three different constraints. Innovative approaches in the context of restricted models continued by the work of Marchand and Strawderman [23] for location families with densities of the form $f_{0}(x-\theta)$. They actually dealt with a lower bound constraint of the form $\theta>a$, while Marchand and Perron [22] extended the result for spherically symmetric distribution under constrained parameter space $\Theta(m)=\left\{\theta \in \mathbb{R}^{p}:\|\theta\| \leq m\right.$ for some fixed $\left.m>0\right\}$. Further Fourdrinier et al. [8]

\footnotetext{
${ }^{*}$ Correspondence to: Mahmoud Afshari (Email: afshar@pgu.ac.ir). Department of Statistics, Persian Gulf University, Bushehr, Iran.
}

ISSN 2310-5070 (online) ISSN 2311-004X (print)

Copyright (C) 2020 International Academic Press 
took the estimation of location parameter into account with restrictions, for spherically symmetric distributions. Fourdrinier and Marchand [9] studied a constraint of the form $\sum_{i=1}^{p} \frac{\left(\theta_{i}-\tau_{i}\right)^{2}}{\sigma^{2}} \leq m^{2}$, with known $\tau_{1}, \ldots, \tau_{p}, \sigma^{2}$, and $m$ for observation $X_{i} \sim N\left(\theta_{i}, \sigma^{2}\right), i=1, \ldots, p$ on spheres of radius $\alpha$ centered at $\left(\tau_{1}, \ldots, \tau_{p}\right)$. More recently Kortbi and Marchand [17] exhibited a truncated linear estimator for the constraint $\|\theta\| \leq m$, in the multivariate normal model and Marchand and Strawderman [24] developed a unified approach for minimax estimation for restricted parameter space. Karamikabir et al. [14] developed a dominant class of Baranchik-type shrinkage estimators under the balance loss function, when the mean vector is restricted to lie in a non-negative hyperplane. Finally Karamikabir and Afshari [15] provided admissible and minimax estimator for $p$-variate normal distribution based on shrinkage soft wavelet estimator.

For more details on this topic, see van Eeden [25], Marchand and Strawderman [21], Silvapulle and Sen [29] and Fourdrinier et al. [10].

Fourdrinier et al. [7] estimated a location parameter vector when some components are non-negative under quadratic loss function.

This short note is the generalization of Fourdrinier et al. [7] under balance loss function.

The paper is outlined as follows:

In Section 2 we consider the spherical distribution under balance loss function. Section 3 provide shrinkage estimation of non-negative mean vector under balance loss function with known covariance. The performance of shrinkage estimations are investigated by a simulation study in Section 4. Finally, In Section 5, we offer some concluding remarks.

\section{Preliminaries}

In this section we consider the spherical distribution. We investigated the natural and Baranchik type of shrinkage estimator under balance loss function. Some examples of spherical distributions are multivariate normal distribution $\left(N_{p}\left(0, \sigma^{2} I_{p}\right)\right), \varepsilon$-contaminated normal distribution and multivariate $t$ distribution.

In Figure 1, the density function and countour plot of some distributions for $\mu=(0,0)^{T}$ and $\sigma^{2} I_{p}=\left[\begin{array}{ll}2 & 0 \\ 0 & 2\end{array}\right]$ is plotted. The contour plot of spherical distribution are circular shape. That's why spherical distribution also called spherically contoured distribution.

We wish to estimate $\theta=\left(\theta_{1}, \ldots, \theta_{p}\right)^{T}$ by $\delta=\left(\delta_{1}, \ldots, \delta_{p}\right)^{T}$ under the balance loss function:

$$
L_{\omega, \delta_{0}}(\theta, \delta)=\omega\left\|\delta-\delta_{0}\right\|^{2}+(1-\omega)\|\delta-\theta\|^{2}, \quad 0 \leq \omega<1 .
$$

where $\delta_{0}$ ia a target estimator. The special case of the balanced error loss function is quadratic loss when $\omega=0$. The balance loss function was introduced by Zellner [31] to reflect two criteria: goodness of fit and precision of estimation. Then the associated risk function with respect to equation (1), will be denoted by $R(\theta, \delta)=E_{\theta}[L(\theta, \delta)]$. In the context of balance loss function, recently Cao [2], $\mathrm{Hu}$ [13], $\mathrm{He}$ and $\mathrm{Wu}$ [12], Zinodiny et al. [32], Peng et al. [27], Cao and He [3] and Zinodiny et al. [33] have been working on the balance loss function.

Assume $(X, U)$ is a $p+k$ random vector having a spherically symmetric distribution around the $p+k$ vector $(\theta, 0), \operatorname{dim} X=\operatorname{dim} \theta=p$ and $\operatorname{dim} U=\operatorname{dim} 0=k$. Further, suppose that the scalar variational component $\sigma^{2}$ is known which will be posed to $X$.

We will propose the result for the cases where a subset of $\theta_{i} \geq 0, i=1, \ldots, p$ are non-negative, i.e., $\theta_{1} \geq 0, \theta_{2} \geq$ $0, \ldots, \theta_{q} \geq 0$ and that $\theta_{q+1}, \theta_{q+2}, \ldots, \theta_{p}$ are unrestricted. Suppose we define $\lambda_{q}(X)$ for $j=1,2, \ldots, q$ as follows:

$$
\lambda_{q}(X)= \begin{cases}-X_{j}, & X_{j}<0 \\ 0, & X_{j} \geq 0\end{cases}
$$

and $\lambda_{q}(X)=0$ if $j>q$.

In this setting, we consider natural and Baranchik type of shrinkage estimator respectively

$$
\delta_{q}(X)=X+\lambda_{q}(X) .
$$



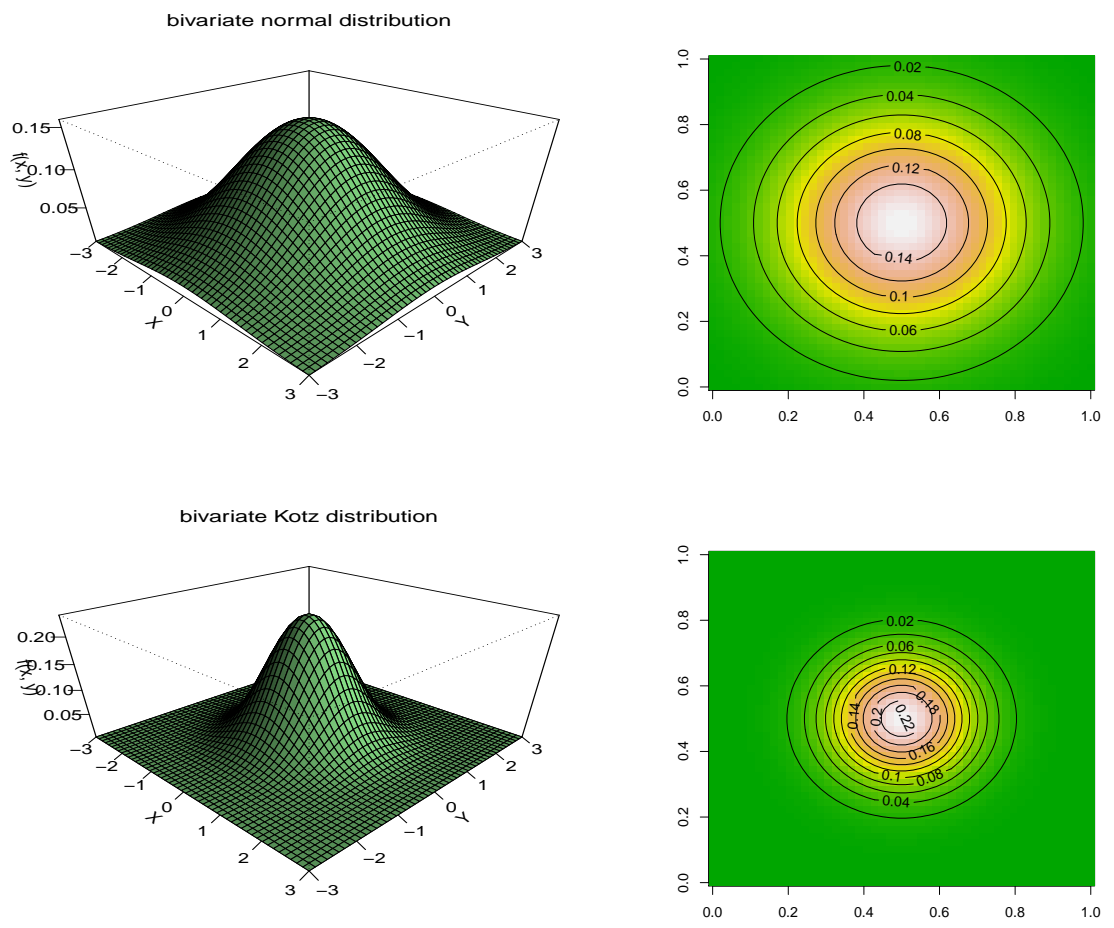

Figure 1. density function and countour plot of some spherical distributions

$$
\delta_{q}(X, U)=X+\lambda_{q}(X)+U^{T} U g(X)
$$

where $g(X)$ is defined as follows:

$$
g(X)=-\frac{c r\left(\|X\|^{2}\right)}{\|X\|^{2}} X
$$

for some constant $c$. Furthermore, suppose that the function $r: \mathbb{R}^{+} \rightarrow[0,1]$ is twice differentiable and concave.

\section{Main Result}

In this section we investigate the domination conditions in order that the specified shrinkage estimators outperform the natural ones in some restrictions. Until the end of the paper, we consider unimodal spherical distributions. We suppose that target estimator $\delta_{0}$ as the following type:

$$
\delta_{0}(X)=X+(1-\omega) \lambda_{q}(X)
$$


We will now be able to express the difference in risk (risk difference) as follows:

$$
\begin{aligned}
\Delta R=\Delta R_{\omega, \delta_{0}}(\theta, \delta)= & R_{\omega, \delta_{0}}\left(\theta, \delta_{q}(X, U)\right)-R_{\omega, \delta_{0}}\left(\theta, \delta_{q}(X)\right) \\
= & E_{\theta}\left[\omega\left(\left\|\delta_{q}(X, U)-\delta_{0}\right\|^{2}-\left\|\delta_{q}(X)-\delta_{0}\right\|^{2}\right)\right. \\
& \left.+(1-\omega)\left(\left\|\delta_{q}(X, U)-\theta\right\|^{2}-\left\|\delta_{q}(X)-\theta\right\|^{2}\right)\right] \\
= & E_{\theta}\left[\omega\left(\left\|X+\lambda_{q}(X)+U^{T} U g(X)-\delta_{0}\right\|^{2}-\left\|X+\lambda_{q}(X)-\delta_{0}\right\|^{2}\right)\right. \\
& \left.+(1-\omega)\left(\left\|X+\lambda_{q}(X)+U^{T} U g(X)-\theta\right\|^{2}-\left\|X+\lambda_{q}(X)-\theta\right\|^{2}\right)\right] \\
= & E_{\theta}\left[\left(U^{T} U\right)^{2}\|g(X)\|^{2}+2(1-\omega) U^{T} U g^{T}(X)(X-\theta)\right. \\
& +2(1-\omega) U^{T} U g^{T}(X) \lambda_{q}(X) \\
& \left.+2 \omega U^{T} U g^{T}(X)\left(X+\lambda_{q}(X)-\delta_{0}\right)\right] .
\end{aligned}
$$

Now by replacing estimator $\delta_{0}(X)$ in (7), we present the notation of risk difference as follows.

$$
\begin{aligned}
\Delta R= & E_{\theta}\left[\left(U^{T} U\right)^{2}\|g(X)\|^{2}+2(1-\omega) U^{T} U g^{T}(X)(X-\theta)\right. \\
& \left.+2(1-\omega) U^{T} U g^{T}(X) \lambda_{q}(X)-2 \omega^{2} U^{T} U g^{T}(X) \lambda_{q}(X)\right] \\
= & E_{\theta}\left[\left(U^{T} U\right)^{2}\|g(X)\|^{2}+2(1-\omega) U^{T} U g^{T}(X)(X-\theta)\right. \\
& \left.+2\left(1-\omega+\omega^{2}\right) U^{T} U g^{T}(X) \lambda_{q}(X)\right] .
\end{aligned}
$$

The second term of the expectation in equation (8), depends on $\theta$. To solve this problem, we use the following lemmas for the multivariate spherical distribution and the special case multivariate normal distribution.

\section{Lemma 1}

For every weakly differentiable function $g: \mathbb{R}^{p} \rightarrow \mathbb{R}^{p}$, for every integer $q$ and for every $\theta \in \mathbb{R}^{p}$ we have

$$
E_{\theta}\left[\left(U^{T} U\right)^{q} g(X)^{T}(X-\theta)\right]=\frac{1}{k+2 q} E_{\theta}\left[\left(U^{T} U\right)^{q+1} \nabla \cdot g(X)\right],
$$

provided these expectations exist.

Proof

See Fourdrinier and Strawderman [5].

\section{Lemma 2}

Suppose that $X \sim N_{p}\left(\theta, \sigma^{2} I_{p}\right)$ and $g: \mathbb{R}^{p} \rightarrow \mathbb{R}^{p}$ with known $\sigma^{2}$, then

$$
E_{\theta}\left[(X-\theta)^{T} g(X)\right]=\sigma^{2} E[\nabla \cdot g(X)] .
$$

Proof

See Stein [28].

Taking $\mathrm{q}=1$ in Lemma 1 , for weakly differentiable function $g$, the risk difference in equation (8) becomes as the following:

$$
\begin{aligned}
\Delta R= & E_{\theta}\left[\left(U^{T} U\right)^{2}\|g(X)\|^{2}+\frac{2(1-\omega)}{k+2}\left(U^{T} U\right)^{2} \nabla \cdot g(X)\right. \\
& \left.+2\left(1-\omega+\omega^{2}\right) U^{T} U g^{T}(X) \lambda_{q}(X)\right] .
\end{aligned}
$$


Lemma 3

If $r$ is a non-negative, differentiable and concave real-valued function, then $r$ is nondecreasing on $\mathbb{R}^{+}$and the function $r(t) / t$ is nonincreasing on $\mathbb{R}^{+}$. Furthermore, if in addition $r$ is twice differentiable, then the function $r\left(\|x\|^{2}\right) /\|x\|^{2}$ is super-harmonic for $p \geq 4$.

Proof

See Fourdrinier et al. [7].

\section{Lemma 4}

Assume $X$ is a real-valued random variable with symmetric unimodal distribution about $\theta \in \mathbb{R}^{+}$. If $f$ is a nonnegative function on $\mathbb{R}^{+}$, then

$$
E_{\theta}\left[f\left(X^{2}\right) X^{2} I_{[X<0]}\right] \leq \frac{1}{2} E_{\theta}\left[(X-\theta)^{2} f\left(X^{2}\right)\right]
$$

Proof

See Fourdrinier et al. [7].

\section{Lemma 5}

If $g$ is a super-harmonic function on $R^{p}$ and $Z$ is a random variable with a uniform distribution on the sphere centered at the origin and of radius $\tau$ then, for any $\theta \in R^{p}$ the expectation $E[g(\theta+Z)]$ is a nonincreasing function of $\tau$.

Proof

See Fourdrinier et al. [7].

\section{Theorem 1}

Suppose $(X, U)$ is a $p+k$ random vector having a spherically symmetric unimodal distribution around the $p+k$ vector $(\theta, 0)$. we assume that scale matrix $\sigma^{2} I_{p}$ under balance loss function. if the following condition holds

1. $k>\frac{4(1-w)(p-2)+2\left(1-\omega+\omega^{2}\right) q}{2(1-w)(p-2)-\left(1-\omega+\omega^{2}\right) q}$,

2. $0<c \leq\left(2(1-\omega) \frac{p-2}{k+2}-\left(1-\omega+\omega^{2}\right) \frac{q}{k-2}\right)$.

Then the shrinkage estimator $\delta_{q}(X, U)$ dominates the natural estimator $\delta_{q}(X)$ for target estimator $\delta_{0}(X)$.

Proof

Since $0 \leq r(\cdot) \leq 1$ is a non-negative, differentiable and concave function by Lemma 3, we have $r^{\prime}(\cdot) \geq 0$, also using Lemma 1 for risk differences $\Delta R^{(1)}$, we have the following inequality:

$$
\begin{gathered}
E_{\theta}\left[c^{2}\left(U^{T} U\right)^{2} \frac{r^{2}\left(\|X\|^{2}\right)}{\|X\|^{2}}-4 c \frac{(1-\omega)}{k+2}\left(U^{T} U\right)^{2} r^{\prime}\left(\|X\|^{2}\right)\right. \\
-2 c(1-\omega) \frac{(p-2)\left(U^{T} U\right)^{2} r\left(\|X\|^{2}\right)}{(k+2)\|X\|^{2}} \\
\left.+2 c\left(1-\omega+\omega^{2}\right)\left(U^{T} U\right) \frac{r\left(\|X\|^{2}\right)}{\|X\|^{2}} \sum_{i=1}^{q} X_{i}^{2} I_{\left[X_{i} \leq 0\right]}\right] \\
\leq E_{\theta}\left[( U ^ { T } U ) ^ { 2 } \frac { r ( \| X \| ^ { 2 } ) } { \| X \| ^ { 2 } } c \left(c-2(1-\omega) \frac{p-2}{k+2}\right.\right. \\
\left.\left.+2\left(1-\omega+\omega^{2}\right) \frac{\sum_{i=1}^{q} X_{i}^{2} I_{\left[X_{i} \leq 0\right]}}{U^{T} U}\right)\right] .
\end{gathered}
$$

Now we suppose that $X_{1}^{q}=\left(X_{1}, \ldots, X_{q}\right), \eta=\left(\theta_{1}, \ldots, \theta_{q}\right), X_{q+1}^{p}=\left(X_{q+1}, \ldots, X_{p}\right)$ and $\mu=\left(\theta_{q+1}, \ldots, \theta_{p}\right)$ also $Z=\sigma^{-1}(X-\theta), V=\left(Z_{1}, \ldots, Z_{q}\right)$ and $T=\left(Z_{q+1}, \ldots, Z_{p}\right)$ in other words $V=\sigma^{-1}\left(X_{1}^{q}-\eta\right)$ and $T=$ 
$\sigma^{-1}\left(X_{q+1}^{p}-\mu\right)$. Since $\|X\|^{2}=\left\|X_{1}^{q}\right\|^{2}+\left\|X_{q+1}^{p}\right\|^{2}$ thus $X_{1}^{q}=\sigma V+\eta$ and $X_{q+1}^{p}=\sigma T+\mu$. Furthermore let $W^{2}=V^{T} V+U^{T} U$, by assuming $\sigma=1$, an upper bound on the conditional expression (10) by Lemma 4 is the following:

$$
\begin{aligned}
& E_{\theta}\left[\left(W^{2}-V^{T} V\right)^{2} \frac{r\left(\left\|X_{1}^{q}\right\|^{2}+\left\|X_{q+1}^{p}\right\|^{2}\right)}{\left\|X_{1}^{q}\right\|^{2}+\left\|X_{q+1}^{p}\right\|^{2}}\right. \\
& \left.\quad \times c\left(c-2(1-\omega) \frac{p-2}{k+2}+\left(1-\omega+\omega^{2}\right) \frac{V^{T} V}{W^{2}-V^{T} V}\right)\right] .
\end{aligned}
$$

Using Lemma $3, \frac{r\left(\left\|X_{1}^{q}\right\|^{2}+\left\|X_{q+1}^{p}\right\|^{2}\right)}{\left\|X_{1}^{q}\right\|^{2}+\left\|X_{q+1}^{p}\right\|^{2}}$ for $p \geq 4$ is super-harmonic and as a result, in $\|X\|^{2}$ is nondecreasing. Hence the conditional risk difference (11) given $W^{2}$ and $T$ is the following:

$$
\begin{aligned}
c E_{\theta} \quad\left[\left(W^{2}-V^{T} V\right)^{2} \frac{r\left(\left\|X_{1}^{q}\right\|^{2}+\left\|X_{q+1}^{p}\right\|^{2}\right)}{\left\|X_{1}^{q}\right\|^{2}+\left\|X_{q+1}^{p}\right\|^{2}}\right. \\
\left.\times\left(c-2(1-\omega) \frac{p-2}{k+2}+\left(1-\omega+\omega^{2}\right) \frac{V^{T} V}{W^{2}-V^{T} V}\right) \mid W^{2}, T\right] \\
\leq \quad c E_{\theta}\left[\left(W^{2}-V^{T} V\right)^{2} \frac{r\left(\left\|X_{1}^{q}\right\|^{2}+\left\|X_{q+1}^{p}\right\|^{2}\right)}{\left\|X_{1}^{q}\right\|^{2}+\left\|X_{q+1}^{p}\right\|^{2}} \mid W^{2}, T\right] \\
\times E_{\theta}\left[\left(c-2(1-\omega) \frac{p-2}{k+2}+\left(1-\omega+\omega^{2}\right) \frac{V^{T} V}{W^{2}-V^{T} V}\right) \mid W^{2}, T\right] .
\end{aligned}
$$

In above inequality we have by covariance inequality and in addition to for fixed $W^{2}$ and $T$, $E_{\theta}\left[\frac{r\left(\left\|X_{1}^{q}\right\|^{2}+\left\|X_{q+1}^{p}\right\|^{2}\right)}{\left\|X_{1}^{q}\right\|^{2}+\left\|X_{q+1}^{p}\right\|^{2}} \mid W^{2}, T\right]$ is nonincreasing in $V^{T} V$ by Lemma 5. Hence, again it suffices to show that the second conditional expectation in equation (12) is nonpositive. On the other hand, since $U^{T} U$ has distribution $\chi_{k}^{2}$ and $V^{T} V$ has distribution $\chi_{q}^{2}$ therefore $\left(V^{T} V\right) / W^{2}$ has distribution $\operatorname{Beta}\left(\frac{q}{2}, \frac{k}{2}\right)$ and we can write as follows:

$$
E\left[V^{T} V /\left(W^{2}-V^{T} V\right)\right]=q /(k-2) .
$$

So the risk difference is non-positive if

$$
0<c \leq\left(2(1-\omega) \frac{p-2}{k+2}-\left(1-\omega+\omega^{2}\right) \frac{q}{k-2}\right) .
$$

A simple calculation shows that the $c$ is positive if and only if

$$
k>\frac{4(1-w)(p-2)+2\left(1-\omega+\omega^{2}\right) q}{2(1-w)(p-2)-\left(1-\omega+\omega^{2}\right) q},
$$

and complete the proof.

As mentioned in the introduction, the multivariate normal distribution $N_{p}\left(\theta, \sigma^{2} I_{p}\right)$ is a member of spherically family distributions. The following result is expressed in relation to multivariate normal distribution.

Corollary 1

Suppose that the distribution is a p-variate normal distribution $N_{p}\left(\theta, \sigma^{2} I_{p}\right)$ with known $\sigma^{2}$. we assume that scale matrix $\sigma^{2} I_{p}$ under balance loss function. if the following conditions are hold: 
1. $p>\frac{\left(1-\omega+\omega^{2}\right) q}{2(1-\omega)}+2$,

2. $0<c \leq\left(2(1-\omega)(p-2)-\left(1-\omega+\omega^{2}\right) q\right)$.

Then the shrinkage estimator $\delta_{q}(X)=X+\lambda_{q}(X)+g(X)$ where $g(X)=-\frac{c \sigma^{2} r\left(\|X\|^{2}\right)}{\|X\|^{2}} X$, dominates the natural estimator $\delta_{q}(X)=X+\lambda_{q}(X)$ for target estimator $\delta_{0}(X)$.

Proof

The proof is similar to Theorem 1, but instead of Lemma 1, Lemma 2 should be used.

\section{Simulation}

In this section we are comparing risk difference between shrinkage estimator and natural estimator in the multivariate normal distribtion.

Risk values ??were obtained from 1000 Monte Carlo replications for $N_{p}\left(\theta, 2 I_{p}\right)$. Shrinkage and natural estimators curve is plotted in Figure 2. Curves are plotted for the target estimators $\delta_{0}(X), p=10$ and for different values of $q$ and $w$.

In Figures, shrinkage estimator curve is below the natural estimator curve. This means that the shrinkage estimator dominates the natural estimator. Since the risk of shrinkage estimator for all $\|\theta\|$ values is less than the risk of natural estimator. As it is shown in Figure 2, with the increasing amount of $w$, the risk difference is increasing and as a result, the shrinkage estimator is better and better than the natural estimator.
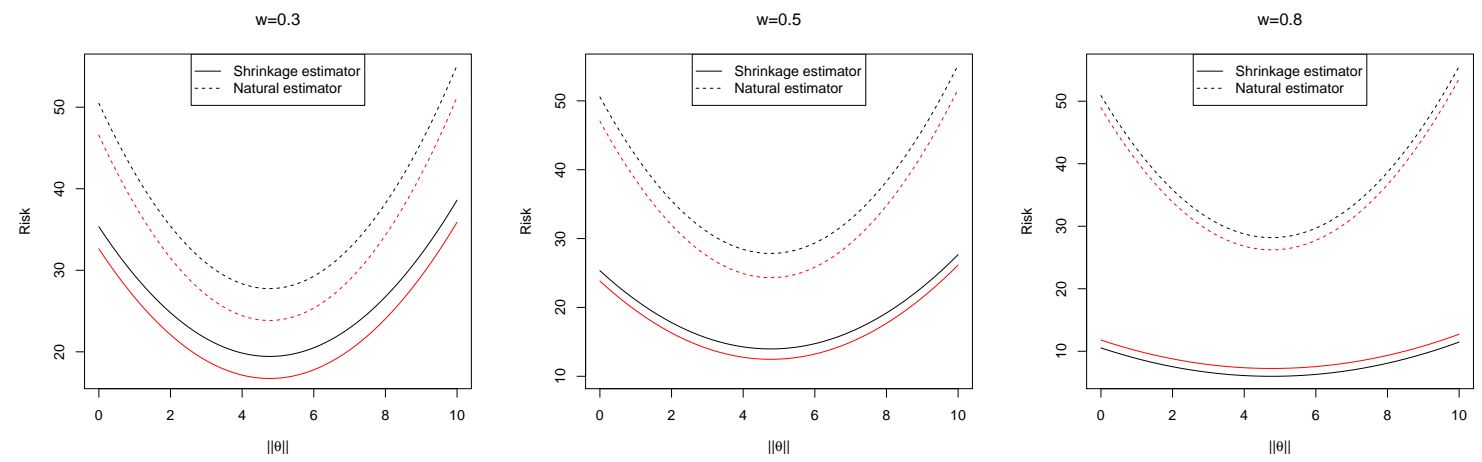

Figure 2. Risk curve for $\delta_{0}(X), p=10$, black line for $q=3$ and red line for $q=7$ for different values of $\omega$.

\section{Conclusion}

In this paper we considered the shrinkage estimator of the location parameter vector for spherically symmetric distributions. According to the risk function, we conclude that the shrinkage estimator has the smaller risk than the natural estimator under the balance loss function, when the covariance matrices are known. The results of the paper are examined by simulation study.

\section{Acknowledgments}

The support of Research Committee of Persian Gulf University is greatly acknowledged. Also the author would like to thank the referees for their constructive suggestions. 


\section{REFERENCES}

1. P. J. Bickel, Minimax estimation of the mean of a normal distribution when the parameter space is restricted, The Annals of Statistics, vol. 9(6), pp. 1301-1309, 1981.

2. M. X. Cao, Admissibility of linear estimators for the stochastic regression coefficient in a general Gauss-Markoff model under a balanced loss function, Journal of Multivariate Analysis, vol. 124, pp. 25-30, 2014.

3. M. X. Cao and D. He Admissibility of linear estimators of the common mean parameter in general linear models under a balanced loss function, Journal of Multivariate Analysis, vol. 153, pp. 246-254, 2017.

4. G. Casella and W. E. Strawderman, Estimating a bounded normal mean, The Annals of Statistics, vol. 9, pp. 870-878, 1981.

5. D. Fourdrinier and W. E. Strawderman, A paradox concerning shrinkage estimators: should a known scale parameter be replaced by an estimated value in the shrinkage factor, Journal of Multivariate Analysis, vol. 59(2), pp. 109-140, 1996.

6. D. Fourdrinier and I. Ouassou, Estimation of the mean of a spherically symmetric distribution with constraints on the norm, Canadian Journal of Statistics, vol. 28(2), pp. 399-415, 2000.

7. D. Fourdrinier, I. Ouassou and W. E. Strawderman, Estimation of a parameter vector when some components are restricted, Journal of Multivariate Analysis, vol. 86, pp. 14-27, 2003.

8. D. Fourdrinier, W. E. Strawderman and M. T. Wells, Estimation of a location parameter with restrictions or vague information for spherically symmetric distributions, Annals of the Institute of Statistical Mathematics, vol. 58, pp. 73-92, 2006.

9. D. Fourdrinier and E. Marchand, On Bayes estimators with uniform priors on spheres and their comparative performance with maximum likelihood estimators for estimating bounded multivariate normal means, Journal of Multivariate Analysis, vol. 101, pp. 1390-1399, 2010.

10. D. Fourdrinier, W. E. Strawderman and M. T. Wells, Shrinkage Estimation. Springer Series in Statistics, 2018.

11. C. Gatsonis and B. MacGibbon and W. E. Strawderman, On the the estimation of a restricted normal mean, Statistics and Probability Letters, vol. 6, pp. 21-30, 1987.

12. D. He and D. Wu, Admissible linear estimators of multivariate regression coefficient with respect to an inequality constraint under matrix balanced loss function, Journal of Multivariate Analysis, vol. 129, pp. 37-43, 2014.

13. G. Hu, G. Li and S. Yu, Optimal and minimax prediction in multivariate normal populations under a balanced loss function, Journal of Multivariate Analysis, vol. 128, pp. 154-164, 2014.

14. H. Karamikabir, M. Afshari and M. Arashi, Shrinkage estimation of non-negative mean vector with unknown covariance under balance loss, Journal of Inequalities and Applications, 2018:331, 2018.

15. H., Karamikabir, and M., Afshari, Wavelet Shrinkage Generalized Bayes Estimation for Elliptical Distribution Parameters under LINEX Loss, International Journal of Wavelets, Multiresolution and Information Processing, vol. 17(1), 2019 Accepted.

16. T. Kariya, Equivariant estimation in a model with an ancillary statistics, Annalls of Statistic, vol. 17, pp. 920-928, 1989.

17. O. Kortbi and E. Marchand, Truncated linear estimation of abounded multivariate normal mean, Journal of Statistical Planning and Inference, vol. 142, pp. 2607-2618, 2012.

18. E. Marchand, Estimation of a multivariate normal mean with constraints on the norm, Canadian Journal of Statistics, vol. 21(4), pp. 359-366, 1993.

19. E. Marchand and N. Giri, James-Stein estimation with constraints on the norm, Communications in Statistics - Theory and Methods, vol. 22(10), pp. 2903-2924, 1993.

20. E. Marchand, On the estimation of the mean of a $N_{p}(\mu, \Sigma)$ population with $\mu^{\prime} \Sigma^{-1} \mu$ known, Statistics and Probability Letters, vol. 21, pp. 69-75, 1994.

21. E. Marchand and W. E. Strawderman, Estimation in restricted parameter spaces: a review, A Festschrift for Herman Rubin, IMS Lecture Notes ,Monogr. Ser, vol. 45, pp. 21-44, 2004.

22. E. Marchand and F. Perron, Improving on the mle of a bounded location parameter for spherical distributions, Journal of Multivariate Analysis, vol. 92, pp. 227-238, 2005.

23. E. Marchand and W. E. Strawderman, Improving on the minimum risk equivariant estimator of a location parameter which is constrained to an interval or a half - interval, Annals of the Institute of Statistical Mathematics, vol. 57(1), pp. 129-143, 2005.

24. E. Marchand and W. E. Strawderman, A unified minimax result for restricted parameter spaces, Annals of the Institute of Statistical Mathematics, vol. 18(2), pp. 635-643, 2012.

25. C. van Eeden, Estimation in restricted parameter spaces- some history and recent developments, CWI Quarterly, vol. 9, pp. 69-76, 1996.

26. F. Perron and N. Giri, On the best equivariant estimator of the mean of multivariate normal population, Journal of Multivariate Analysis, vol. 32, pp. 1-16, 1989.

27. P. Peng, G. Guikai Hu, and J. Liang, All admissible linear predictors in the finite populations with respect to inequality constraints under a balanced loss function, Journal of Multivariate Analysis, vol. 140, pp. 113-122, 2015.

28. C. M. Stein, Estimation of the mean of a multivariate normal distribution, The Annals of Statistics, vol. 9(6), pp. 1135-1151, 1981.

29. M. J. Silvapulle and P. K. Sen, Constrained Statistical Inference Inequality, Order, and Shape Restrictions, John Wiley and Sons, Inc., Hoboken, New Jersey, 2005.

30. A. T. K. Wan, G. Zou and A. H. Lee, Minimax and $\Gamma$-minimax estimation for the Poisson distribution under LINEX loss when the parameter space is restricted, Statistics and Probability Letters, vol. 50, pp. 23-32, 2000.

31. A. Zellner, Estimation of the mean of a multivariate normal distribution, Bayesian and non-Bayesian estimation using balanced loss functions. In: Berger, J.O., Gupta, S.S. (Eds.). Statistical Decision Theory and Methods. Springer Verlag, New York, 337-390, 1994.

32. S. Zinodiny, S. Rezaei and S. Nadarajah, Bayes minimax estimation of the multivariate normal mean vector under balanced loss function, Statistics and Probability Letters, vol. 93, pp. 96-101, 2014.

33. S. Zinodiny, S. Rezaei and S. Nadarajah, Bayes minimax estimation of the mean matrix of matrix-variate normal distribution under balanced loss function, Statistics and Probability Letters, vol. 125, pp. 110-120, 2017. 